\title{
Citoyenneté, UNiversalisme ET COSMOPOLITISME STOÏCIENS : LE CAS ROMAIN \\ Citizenship, universalism AND STOIC \\ COSMOPOLITANISM: THE ROMAN CASE
}

\author{
Antonio Gonzales \\ Université de Franche-Comté \\ ANTONIO.GONZALES@UNIV-FCOMTE.FR
}

\section{RÉSUMÉ}

La question de la citoyenneté a très vite dépassé dans le cadre de l'empire territorial romain le problème du simple statut juridique pour poser la question de l'articulation philosophique et politique d'une citoyenneté en quelque sorte déterritorialisée puisqu'être citoyen romain ne signifie plus forcément habiter Rome ou venir exercer ses droits civiques à Rome.

L'extension territoriale et l'intégration plus ou moins rapide des populations a suscité une réflexion sur le rapport entre l'individu citoyen et le groupe civique désormais dispersé à l'échelle de l'empire. S' il existe des citoyens romains sur l'ensemble territorial de l'empire, la citoyenneté est-elle simplement une citoyenneté romaine qui se répand dans l'espace impérial en conservant la centralité romaine ou, au contraire, est-elle en train d'acquérir une spécificité

\section{Abstract}

The question of citizenship very quickly went beyond the problem of simple legal status within the Roman territorial empire to raise the question of the philosophical and political articulation of a somewhat deterritorialized citizenship, since being a Roman citizen no longer necessarily means living in Rome or coming to exercise your civil rights in Rome.

The territorial extension and the more or less rapid integration of populations has prompted reflection on the relationship between the individual citizen and the civic group now dispersed throughout the empire. If there are Roman citizens throughout the empire, is citizenship simply a Roman citizenship that spreads throughout the imperial space while preserving Roman centrality or, on the contrary, is it acquiring such a specificity that 
telle qu'elle peut être comprise comme une citoyenneté supra-civique qui acquiert un caractère universel tout en gardant ces spécificités initiales ou alors devient-elle une citoyenneté qui se substitue à l'idée même du civique? Les débats sur ces mutations potentielles ont agité les juristes, les philosophes et les hommes politiques entre République et Empire. it can be understood as a supra-civic citizenship that acquires a universal character while retaining its initial specificities or does it become a citizenship that replaces the very idea of the civic? The debates on these potential changes have stirred up lawyers, philosophers and politicians between the Republic and the Empire.

\section{MOTS CLÉ}

Citoyenneté, Rome, Universalisme, Cosmopolitisme, Stoïcisme

\section{KEYWORDS}

Citizenship, Rome, Universalism, Cosmopolitanism, Stoicism 
LES ROMAINS SE SONT INTERROGÉS TRÈS TÔT SUR L'APPARTENANCE à un groupe identifié comme porteur de valeurs particulières sur le plan civique et culturel auquel on pouvait agréger d'autres individus. Les problèmes posés par la diffusion de la citoyenneté romaine vont les contraindre à évaluer la valeur intégrative, universelle ou cosmopolite de celle-ci. Si, durant la période républicaine, la conquête de la Péninsule italienne et du Bassin méditerranéen pose la question de la citoyenneté de manière collective plutôt qu'à titre individuel en raison de l'intégration de peuples entiers dans l'empire, la création du Principat et la stabilisation des frontières conduisent progressivement à envisager la citoyenneté romaine comme une potentielle citoyenneté commune (communis patria) aux hommes libres à laquelle on pourra parvenir soit par étapes, soit par l'exercice des magistratures supérieures dans les cités, soit encore en fonction d'une décision juridique de l'empereur.

Il n'est pas question d'aborder ici l'histoire de la citoyenneté romaine et de sa diffusion dans le temps et dans l'espace durant près d'un millier d'années. Il s'agit plutôt de mener une réflexion sur les circonstances intellectuelles, sociales et politiques qui ont conduit non seulement à la possibilité d'une diffusion de la citoyenneté romaine, mais aussi à la définition progressive d'une citoyenneté romaine comme aboutissement de l'intégration. Les hommes libres peuvent-ils alors constituer une communauté unique soudée par la ciuitas romana en oubliant leurs origines diverses? Un cosmopolitisme romain est-il possible ? Nous allons essayer ci-après de donner quelques pistes de réflexion qui ne peuvent, en aucun cas, avoir la prétention à l'exhaustivité.

Si la question de la nature et du sens du cosmopolitisme est déjà ancienne dans l' histoire de la pensée grecque lorsqu'elle se pose à Rome, puisque la naissance

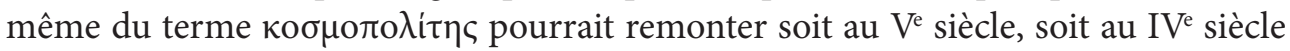
avant notre ère si l'on fait abstraction d' une possible origine héraclitéenne du terme, ${ }^{1}$ l'émergence et la constitution d'un empire territorial à l'échelle méditerranéenne vont

1. Brown, 2006, pp. 549-558 ; Long, 2008, pp. 50-58 ; Konstan, 2009, pp. 473-484. 
confronter Rome à la question du changement d'échelle pour le contrôle et la gestion de ce vaste empire. L'idée d'une association des vaincus s'impose d'abord pour les élites puis, plus largement, pour les populations libres des provinces. Si cette association se fait, au fil des siècles, selon une stratégie qui maintient l'essentiel des citoyens dans une citoyenneté "secondaire » qui est la citoyenneté latine ou en marge de la romanité avec le statut de pérégrin, les promotions civiques finissent par poser la question du rapport entre citoyenneté romaine et citoyenneté latine et de leur imbrication juridique et politique. Si ces deux formes de citoyenneté restent " terrestres "

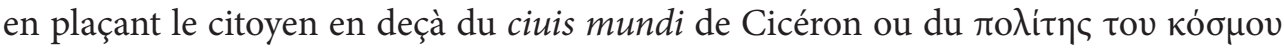
d'Épictète, il n'est pas impossible que la citoyenneté romaine ait été pensée progressivement comme l'équivalent d'une forme supérieure de citoyenneté, ce qu'elle était effectivement juridiquement et politiquement. Cicéron, dans les Lois, en donne d'ailleurs une explication qui s'adresse à l'homme sage : «cet homme, quand il aura observé à fond le ciel, la terre, les mers et la nature universelle ; qu' il verra d'où viennent toutes ces choses, où elles doivent aller, quand, comment elles périrent, ce qu'il y a en elles de mortel et de fragile, ce qu' il y a de divin et d'éternel ; quand il saisira pour ainsi dire le dieu qui en est le régulateur et le maître, qu'il reconnaîtra qu'il n'est plus borné par les murs d'une cité, habitant d'une région limitée, mais concitoyen du monde entier comme d'une ville unique (sed ciuem totius mundi quasi unius urbis agnouerit) ; au milieu de toute cette grandeur, dans cette vision et cette connaissance de la nature, ô dieux immortels, comme il prendra conscience de lui-même ! Comme il dédaignera, regardera de haut et tiendra pour néant les choses que la masse appelle les plus grandes! ». ${ }^{2}$ Rédigé sans doute lors de l'été 52 à la suite du de Republica, il fait ici un point sur les droits des «concitoyens " à un moment où il est tenu à l'écart des grandes responsabilités politiques. Cette réflexion qui consolide l'idée d'un droit naturel reprend l'idée partagée par les stoïciens d'une raison qui est la Loi naturelle d'une cité commune aux hommes et aux dieux ${ }^{3}$ ayant aboli les " murs", privilège des cités libres, de la cité terrestre pour se fondre dans une cité unique. Cette dernière idée est particulièrement mise en avant par Arius Didyme, l'un des précepteurs du futur Auguste. Ce dernier fonde le Principat avec l'objectif de réunir les hommes autour et sous la tutelle du Princeps garant terrestre de l'ordre cosmique.

L'idée du cosmopolitisme à Rome a parfois été interprétée comme instrumentale, puisqu'elle n'aurait servi qu'à légitimer sa domination sur son empire en y

2. Cic., Leg. I 61. Trad. de Plinval.

3. Diels, Dox. Graec., frag. 29.

ARYS, 16, 2018 [19-45] ISSN 1575-166X 
associant les élites au détriment de la masse de la population libre. ${ }^{4}$ Cette question est encore à l'ordre du jour, lorsque Marc-Aurèle s' interroge : " si l' intelligence nous est commune, la raison, qui fait de nous des êtres raisonnables, nous est commune. Ceci admis, nous est de même commune cette raison dont c'est le rôle de prescrire ce qu'il faut faire ou non. Ceci admis, la loi aussi nous est commune. Ceci admis, nous sommes concitoyens. Ceci admis, nous faisons partie d'un même corps politique commun. Ceci admis, le monde est comme une cité. De quel autre corps politique commun dira-t-on, en effet, que tout le genre humain fait partie ? C'est de là-haut, de cette cité commune, que nous viennent l' intelligence elle-même, la raison et la loi ; sinon, d'où viendraient-elles ? ». ${ }^{5}$ Pense-t-il aux sages, aux membres des élites civiques ou à l'ensemble des hommes ? Les stö̈ciens ${ }^{6}$ envisageaient le cosmopolitisme comme le moyen normatif d' inclure les sages dans une même conception de l'être et du mode de vie universel soumis à une même loi comme les membres d'un troupeau paissant ensemble. Plutarque avait déjà moqué, dans La fortune ou la vertu d'Alexandre, « la République, tant admirée, de Zénon, fondateur de l'École stoïcienne, [qui] tend en somme vers un seul but; à ce que nous ne vivions plus séparés en cités ou en communautés régies par des lois différentes, à ce que nous considérions l'humanité tout entière comme une seule communauté politique, à ce qu'il n'y ait plus qu'un mode de vie, qu'un ordre unique, comme d'un grand troupeau vivant sur le même pâturage. Zénon, dans son ouvrage, a donné forme au rêve confus d'une constitution parfaite fondée sur la philosophie... ». ${ }^{7}$ Pourtant, l'idée d'une loi commune à tous (кoเvós vó $\boldsymbol{\mu o s}$ ) qui s'exprimait chez Cicéron, philosophe non stoïcien, à travers l'idée d'humanitatis societas ${ }^{8}$ et de commune humanitatis ius ${ }^{9}$ laissait entrevoir une

4. Diogène de Sinope lorsqu'il s'était qualifié de citoyen de l'univers - si nous accordons foi à nos sources - englobait semble-t-il sinon l'ensemble des hommes du moins la totalité des citoyens et non une fraction seule.

5. M. Aur., Med. IV 4, 1-5. Trad. Trannoy.

6. Dont Zénon de Kition s'il a réellement soutenu l'idée d'un cosmopolitisme. Sur Zénon et les «Zenoniens ", cf. Shaw, 1985, p. 21 qui accorde une place importante à l'origine géographique et au statut juridique des premiers maîtres de la Stoa.

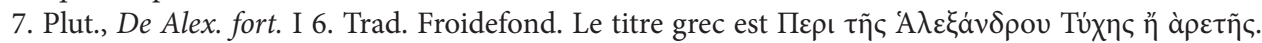
Épictète (Arr., Epict. diss. II 14, 24-29) qui utilise l'idée de troupean et celle de grand marché aux idées : "Ainsi en est-il dans cette foire du monde où nous vivons... ». Trad. Souilhé. Cf. Shaw, 1985, pp. 20-21 et 28 , n. 24. Sur l'idée de "pâturage commun ", voir Africa, 1959, p. 463, nn. 2 et 4 qui voit un calembour entre vómoc (loi) et vouóc (pâturage). Sur la transmission du cosmopolitisme de Zénon à travers Plutarque jusque vers Kant et Habermas, voir Pagden, 2000, pp. 3-22.

8. Cic., Rep. II 26.

9. Cic., Fin. II 24. 
évolution vers l'idée d'un monde commun régi par une même loi sans nier les cités concrètes, terrestres et historiques. Sénèque rappellera : «[...] qu'il y a deux républiques : l'une, grande et vraiment publique (commune, universelle), embrasse les dieux et les hommes; nous ne nous y confinons pas dans tel ou tel coin particulier, et la cité que nous habitons n'a de bornes que celles du soleil ; l'autre, celle à laquelle nous attache le hasard de la naissance (ce sera Athènes, Carthage ou n'importe quelle autre ville), ne comprend plus tous les hommes, mais un groupe d' hommes déterminé. Il y a des gens qui donnent leurs soins à la fois aux deux républiques, à la grande et à la petite; d'autres seulement à la petite, d'autres seulement à la grande. Cette grande république, nous pouvons la servir même dans l'oisiveté, et mieux peut-être dans

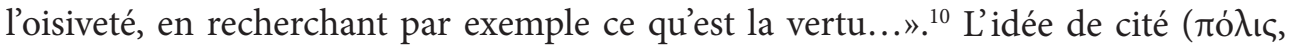
ciuitas) n'est pas une idée obsolète. Elle se renforce par le déploiement de multiples formes statutaires à travers l'empire comme l'a pensé Aulu-Gelle à propos des colonies projection de Rome lorsqu' il écrit dans ses Nuits attiques que les Coloniae quasi [populi romani] effigies paruae simulacraque esse quaedam uidentur. ${ }^{11}$ Les colonies ne sont pas Rome et pourtant elles portent en elles tous les ingrédients de la romanité : enceinte, forum, basilique, capitole, institutions romaines, citoyenneté, etc. L'uniformisation urbaine à travers l'empire renforce l'idée d'une appartenance commune à un même ensemble y compris chez les Grecs à compter du II siècle de notre ère du moins dans les éloges de Rome dont on ne pouvait attendre moins.

L'idée d'un monde commun dominé par Rome qui diffuse ses valeurs a pris forme progressivement. Tout aussi progressivement progresse l'idée d'un destin commun de Rome et des parties de son empire. Ce n'est pas du cosmopolitisme, mais le cadre territorial de l'empire favorise l'émergence d'un sentiment d'appartenance à

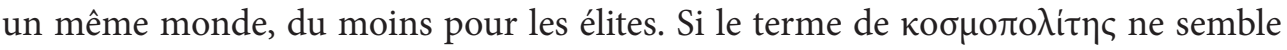
plus être utilisé par les cyniques et les stoïciens des $\mathrm{II}^{\mathrm{e}}$ et $\mathrm{I}^{\mathrm{er}}$ siècles avant notre ère, cela ne veut pas dire que l'idée n'ait pas été discutée dans les écoles philosophiques ou dans certains cercles politiques comme celui des Scipions à Rome. ${ }^{12}$ De même, si les stoïciens sont mentionnés à Rome avec l'ambassade de 155 avant notre ère, ${ }^{13}$ de quoi parlait-on vraiment dans ces discussions? Les divergences philosophiques ont-elles été tues dans la perspective de la défense des intérêts d'Athènes auprès des

10. Sen., De otio IV 1-2. Trad. Waltz.

11. Gell., NA XVI 13, 9.

12. Shaw, 1985, p. 27 qui cite Finley, 1975b, p. 199.

13. Cette ambassade réunit l'académicien Carnéade et le péripatéticien Critolaus permet à Diogène de Babylone de diffuser les thèses stoïciennes auprès du Sénat lorsqu' ils plaident la cause d'Athènes accusée de pillage par Oropos. 
élites romaines ? Cette ambassade a-t-elle été aussi l'occasion d'envisager une nouvelle conception des rapports entre le modèle poliade et Rome ? Si Carnéade fait scandale en critiquant l'impérialisme romain, Critolaos et Diogène de Babylone séduisent l'aristocratie romaine en diffusant leur philosophie sans doute beaucoup plus conservatrice et favorable au maintien des structures sociales et politiques en place. La crise du système de la $\pi$ ó $\lambda ı$ c et les tensions sociales internes aux cités grecques ont dû interpeller les interlocuteurs romains dont Scipion Émilien. Les tensions sociales, politiques et militaires à Rome, dans les décennies 150 à 130, vont favoriser l'émergence de discussions et de propositions alternatives y compris stö̈ciennes que les débats philosophiques ont dû nourrir. Sur le plan factuel, selon Iseltreut Hadot, c'est une divergence d'interprétation sur le régime lacédémonien qui aurait été la base du conflit, en 134, entre les Gracques soutenus par les Scaevolae ainsi que Blossius de Cumes et Scipion Émilien, représentant du parti conservateur dont un des proches est Panétius. ${ }^{14}$ Un autre élément de controverse entre stoïciens reposait, selon Diogène Laërce, sur l'interprétation par Sphairos du sens à donner à l'idée de la cité cosmique dans Sur la constitution politique des Lacédémoniens. ${ }^{15}$ Ce texte aurait inspiré Agis et Cléomène et conduit à une réponse aux problèmes liés à la citoyenneté à Sparte si l'on en croit les Vies de Plutarque, mais les problèmes étaient sans doute plus liés à la question de la propriété et du service militaire. ${ }^{16}$ Or, un siècle plus tard, parallèlement aux problèmes sociaux et à ceux de la propriété de la terre auxquels les Gracques vont tenter de répondre, se pose à Rome la question de la diffusion de la citoyenneté. Cette dernière, romaine complète (ciuitas Romana optimo iure), incomplète (sine suffragio) ou latine, est pensée par Rome en fonction de ses intérêts directs, malgré une évolution des structures politiques et territoriales vers une uniformisation qui ne sera réelle pour l' Italie qu'après la Guerre sociale (91-89) et la diffusion par Rome de la citoyenneté romaine à tous les Italiens. Il faudra attendre, par ailleurs, 49 avant notre ère pour que toute la péninsule soit couverte par la citoyenneté romaine, alors même que Rome expérimente dans le cadre provincial, occidental essentiellement, une nouvelle forme de propédeutique civique avec le modèle de la citoyenneté latine (ciuitas Latina per honorem). Cette diffusion de la citoyenneté romaine et des étapes qui peuvent y mener n'est pas sans poser des problèmes politiques, mais aussi juridiques et philosophiques sur l'articulation entre les appartenances locales et romaine.

14. Dudley, 1941, pp. 95-99 ; Becker, 1964, pp. 125-134 ; Hadot, 1970, pp. 133-179; Shaw, 1985, pp. 45-46 ; Nicolet, 1965, pp. 142-159.

15. Diog. Laert., VII 178. Trad. R. Goulet. Le troisième livre est cité par Athénée, SVF I 630.

16. Plut., Cleom. 2, 2-3; 10, 11; 11, 4 ; Agis 2, 7. Sur les liens entre les stoïciens et les mouvements réformateurs, voir Shaw, 1985, p. 27, n. 20 et p. 45 ; Africa, 1959, pp. 461-469. 
C'est à ce défi que doit répondre Rome en pratiquant, à travers la gestion impériale, une politique de la hiérarchie des citoyennetés en plaçant au sommet la citoyenneté romaine sans nier les modalités civiques locales, ce que les historiens Jane Burbank et Frederick Cooper ont qualifié, à partir d'une étude globale sur les empires, de " politic of difference ». Pour ces derniers, " dans certains empires, la politique de la différence fut synonyme de reconnaissance, dans la multiplicité des populations et la diversité de leurs coutumes, d'une réalité ordinaire de l'existence ; dans d'autres, elle servit à tracer des frontières strictes entre les indifférenciés de l'intérieur et les "barbares " de l'extérieur $» .^{17}$ De toute évidence, la politique romaine sur la longue durée relève de la deuxième catégorie. En effet, on peut aisément comprendre que Rome, sur un spectre impérial de plus d'un millier d'années, eut pour pratique de rapprocher le statut politique des individus en séduisant d'abord les élites. Cette homogénéisation des points de vue acquise pour les élites conduisit à une extension territoriale et démographique de la citoyenneté romaine et de sa diffusion. La généralisation progressive de la citoyenneté latine jusqu'en 212 de notre ère et l'édit de Caracalla conditionnèrent culturellement les habitants de l'empire pour l'acquisition de la citoyenneté romaine, à l'exclusion des Barbares, des déditices et des esclaves résidant sur le sol romain. Puisque sous l'Empire et jusqu'à Caracalla, les élites locales peuvent accéder à la citoyenneté romaine grâce au ius ciuitatis per magistratum adipiscendae, l'exercice des magistratures locales sur le modèle de celles de Rome va constituer un parcours civique et un apprentissage de la Romanitas. Rome maintient ainsi les élites provinciales et favorise l'émergence d'une hérédité fonctionnelle et sociale aussi bien en Occident qu'en Orient, rapprochant, malgré les différences structurelles et culturelles, les deux principaux pôles de l'empire. De ce point de vue, ce ne sont pas les élites provinciales qui ont voulu devenir romaines, c'est Rome qui a décidé de s'appuyer sur les élites locales pour adapter les institutions au modèle romain et à la domination de l' Vrbs. ${ }^{18}$ Il n'y a donc de cosmopolitisme possible, d'après ce schéma, que pour les élites. Le reste de la population y compris lorsqu'elle est libre en est de fait exclu politiquement mais pas culturellement, au même titre que les étrangers, les esclaves, etc.

L'assimilation des élites et la subordination des populations a été la première étape de la construction de l'idée d'un pouvoir impérial " cosmopolite ", ce qui ne fait pas du cosmopolitisme romain une dévalorisation de la citoyenneté locale, mais plutôt une extension du champ de la citoyenneté romaine à l'empire. Toutefois, les

17. Burbank et Cooper, 2011, p. 28.

18. Voir la discussion dans Ando, 2016, pp. 182-185 ; contra Brunt, 1976, pp. 161-173.

ARYS, 16, 2018 [19-45] ISSN 1575-166X 
réflexions théoriques sur la citoyenneté mondiale d'un Cicéron ou d'un Épictète s'ap-

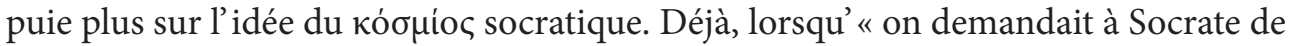
dire où il était citoyen. "Citoyen du monde " fut la réponse : c'est le monde entier en effet qu' il considérait comme son pays d'origine et sa cité « mundanum » inquit; totius enim mundi se incolam et civem arbitrabatur $" .{ }^{19}$ Être citoyen du monde relève

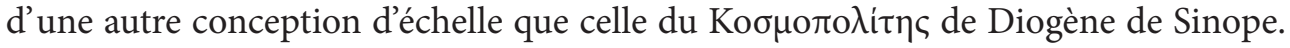
Cicéron utilise d'ailleurs le terme mundanus, non attesté avant lui me semble-t-il, pour transposer en latin le terme socratique кó $\sigma \mu$ íoc. Cette conception est reprise par

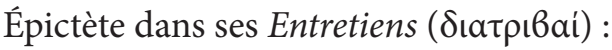

«S'il est vrai qu'il y a une parenté entre Dieu et les hommes, comme le prétendent les philosophes, que reste-t-il à faire aux hommes, sinon d'imiter Socrate, c'est-à-dire de ne jamais répondre à qui leur demande quel est leur pays : «Je suis citoyen d'Athènes ou citoyen de Corinthe, mais je suis citoyen du monde? " Pourquoi se dire, en effet, Athénien, plutôt que simplement de ce coin de terre où ton pauvre corps a été jeté à sa naissance? N'est-il pas clair que tu dois ton nom à une origine plus importante, qui embrasse non seulement ce coin de terre, mais encore ta maison tout entière, et, en un mot, le pays où tes ancêtres se sont perpétués jusqu'à toi, d'où vient que tu peux t'appeler Athénien ou Corinthien ? Si l'on s'est donc rendu compte de l'organisation de l'univers, si l'on a compris que, «de toutes les choses, la principale, la plus importante, la plus universelle, c'est le système composé de Dieu et des hommes, que de là proviennent les semences génératrices non seulement de mon père ou de mon grand-père, mais de tout ce qui sur terre a vie et croissance, spécialement des êtres raisonnables, car seuls, par nature, ils participent à la société divine, liés qu'ils sont à Dieu par la raison ", - pourquoi ne se dirait-on pas citoyen du monde ? Et pourquoi pas fils de Dieu ? Pourquoi redouter les vicissitudes humaines quelles qu'elles soient? Mais la parenté avec César ou avec quelque puissant à Rome suffira à permettre de vivre en sûreté, de se mettre au-dessus de tout mépris, au-dessus de toute crainte, et le fait d'avoir Dieu pour créateur, pour père, pour protecteur, ne pourrait donc suffire à nous délivrer des chagrins et des terreurs?

- «Et comment mangerai-je, dit-on, si je n'ai rien?»

- Et comment font les esclaves, comment font les fugitifs ? En quoi placent-ils leur confiance quand ils quittent leurs maîtres? En leurs champs, en leurs serviteurs, en leur argenterie ? En rien de tout cela, mais en eux-mêmes ${ }^{20}$

19. Cic., Tusc. V 108. Trad. Humbert ; cf. Diog. Laert., VI 63.

20. Arr., Epict. Diss. I 9, 1-6. Trad. Souilhé. 
La conception développée par Épictète ne condamne pas le pouvoir et n'exclut pas l'empereur rendant ainsi le cosmopolitisme compatible avec l'empire, patrie commune de tous les hommes qui considèrent qu' ils sont les citoyens de la cité de Zeus comprenant hommes et dieux. Certes, mais qu'est-ce que le bon gouvernement d'une cité, d'un royaume ou d'un empire ? Celui d'un roi, d'un empereur conseillé par un stoïcien ou empreint de stoïcisme lui-même ? D’emblée la question du régime démocratique pourrait sembler incongrue puisque pour les stoïciens seuls les sages doivent gouverner. Or, si le propre d'une démocratie est de confier la gestion au choix du plus grand nombre constitué d'insensés pour un stoïcien, elle serait donc le régime le moins à même de convenir. Si le stoïcisme reconnaît à chaque individu la possibilité d'atteindre la vertu et si, pour Zénon, seuls les sages sont citoyens, ${ }^{21}$ Épictète précise néanmoins à propos du sage :

« tu es citoyen du monde et partie de ce monde, non pas une des parties subordonnées mais une des parties dominantes; en effet tu es capable de suivre et de comprendre l'administration divine et de réfléchir à ses conséquences. Quelle est donc la profession du citoyen ? De n'avoir aucun intérêt privé, de ne délibérer sur rien comme s'il était isolé, mais plutôt de la manière suivante : si la main ou le pied étaient doués de faculté de raisonner, qu' ils comprenaient et suivaient l'ordre de la nature, jamais ils ne se mettraient en mouvement ou ne désireraient autrement qu'en se rapportant au tout $\gg .{ }^{22}$

On notera que pour Épictète la citoyenneté est celle du Tout et non celle d'une cité quelconque. Dans ce contexte, le régime politique de la cité est affaire de circonstances auxquelles la cité démocratique ne peut répondre que partiellement et imparfaitement eu égard aux rapports entre petite cité et cité absolue. La démocratie

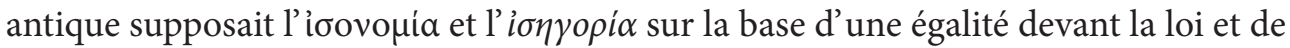
compétence des citoyens. Or, si homme, femme, esclave peuvent accéder à la vertu, ce n'est pas pour autant qu'ils sont sages : ils demeurent pour le plus grand nombre des insensés incapables de distinguer le bien du mal, ce que Laélius précise dans le $D e R e$ publica de Cicéron de la manière suivante :

« ... il existe certes une vraie loi, c'est la droite raison ; elle est conforme à la nature, répandue chez tous les hommes; elle est immuable et éternelle ; ses ordres appellent au devoir ; ses interdictions détournent de la faute. Si toutefois elle n’adresse jamais en vain aux honnêtes gens ses ordres et ses interdictions, elle ne peut, par ces moyens,

21. Diog. Laert., VII 33.

22. Arr., Epict. Diss. II 10, 1-11. Trad. Souilhé, citée par Veillard, 2017, p. 11 ; Shaw, 1985, pp. 29-30.

ARYS, 16, 2018 [19-45] ISSN 1575-166X 
faire impression sur les malhonnêtes. C'est un sacrilège que de la remplacer par une loi contraire ; il est interdit de n'en pas appliquer une seule disposition; quant à l'abroger entièrement, personne n'en a la possibilité. Ni le sénat, ni le peuple ne peuvent nous soustraire à l'autorité de cette loi ; il est inutile de chercher un Sextus Ælius pour l'expliquer et l' interpréter; elle sera la même à Rome et à Athènes; la même maintenant et plus tard. Bref, cette loi unique, éternelle et immuable s' imposera à toutes les nations et à tous les temps, et un seul dieu commun à tous sera comme l'éducateur et le chef de tous. C’est lui qui a fait cette loi, qui l'interprète et nous l'a proposée. L' homme qui refusera de lui obéir devra se fuir lui-même et, comme il a méprisé la nature humaine, il subira les plus cruels châtiments, même au cas où il aurait échappé à tout ce que l'on considère comme un supplice ${ }^{23}$

Un autre point doit être précisé. Contrairement à une idée assez diffusée, le cos-

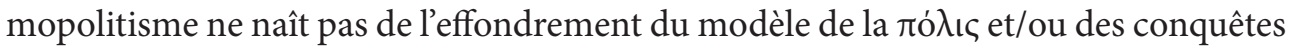
d'Alexandre. ${ }^{24}$ Présente dans la pensée présocratique ${ }^{25}$ et, comme le pense E. Brown, à propos de la base intellectuelle de la philosophie de Diogène de Sinope, chez Socrate lui-même bien qu' il reconnaisse la prévalence des lois civique d'Athènes, ${ }^{26}$ le cosmopolitisme ne serait pas non plus une spécificité cynique, mais aurait été partagé par les philosophies présocratique et stoïcienne. L'épicurisme de l'époque hellénistique, comme le précise A.A. Long, ${ }^{27}$ aurait été peu sensible à cette dimension. L'amplitude de la pensée cosmopolite montre en tout cas l'importance de cette idée que Rome va reprendre en tant que discours " universaliste » et " cosmopolite».

$\mathrm{Si}$ « universalisme » et « cosmopolitisme » ne sont pas synonymes, les notions d' « empire » et de « cosmopolitisme » ne le sont pas plus. Si les empires ont besoin de développer un discours et des outils d'échange pour fonder une communauté " cosmopolite », il existe d'autres formes de réseaux qui visent une forme de cosmopolitisme qui ne passe pas par un pouvoir de nature impériale. Les élites impériales qui finissent par se penser et se proclamer cosmopolites attendent des élites locales qu'elles adoptent les modes de vie et de pensée du cosmopolitisme qui légitime à la fois la domination des élites impériales et l'adaptation des populations locales aux modes de vie et de pensée promus par ces mêmes élites. Rome, temple du monde en-

23. Cic., Rep. III 22, 33. Trad. Bréguet.

24. Pour une lecture " positive " de l'enthousiasme de Plutarque vis-à-vis du cosmopolitisme d'Allexandre, voit Konstan, 2009, pp. 479-480.

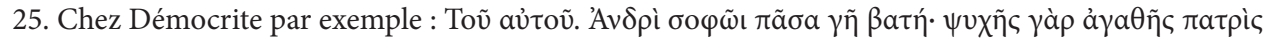

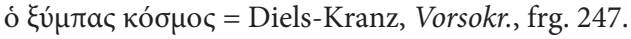

26. Lana, 1951, pp. 196-216 et 317-338; Brown, 2006, pp. 549-552 ; Long, 2008, p. 53.

27. Long, 2008, p. 58. 
tier, ${ }^{28}$ utilise ainsi la religion pour infléchir les comportements et favoriser une fusion progressive des cultures en exportant ses dieux, en accueillant des dieux étrangers et en développant à partir de la mise en place du Principat le culte impérial qui doit favoriser et favorisa l'image de l'empereur comme fédérateur des diversités locales qui ne sont pas abolies mais qui se retrouvent dans la figure de l'empereur, vecteur commun, rendant ainsi possible l'émergence d'une communauté cosmopolite. ${ }^{29}$ Selon cette conception, Plutarque fit d'Alexandre le premier " empereur » cosmopolite..$^{30}$ Marc-Aurèle reprend quant à lui l'idée déjà développée chez Cicéron d'une double appartenance en changeant l'échelle de référence, celle de l'origo et de la communis patria. Dans les deux cas, le cosmopolitisme suppose donc la détention préalable de la citoyenneté. De fait, la question du cosmopolitisme est recoupée par celle de la dimension spirituelle qui la fonde, car il y a une différence importante entre la conception " universelle » socratique de la citoyenneté et celle « cosmopolite» de Diogène. Épictète comme Cicéron célèbre la définition socratique moins dangereuse à ses yeux que celle de Diogène qui apparaît comme trop " révolutionnaire » dans le cadre politique et religieux de la cité comme dans celui de l'espace impérial de Rome. Si celle de Socrate n'est pas incompatible avec la structure impériale et une conception verticale du pouvoir, celle des Cyniques ouvre la voie à une conception plus horizontale, peutêtre plus démocratique, mais de fait assez incompatible avec des institutions qui sont en train d'évoluer vers une monarchie de type universaliste que l'empereur synthétise dans sa personne et dans ses pouvoirs. ${ }^{31}$

L'empereur devient l'élément clef de la cohésion de l'ensemble parce qu'il

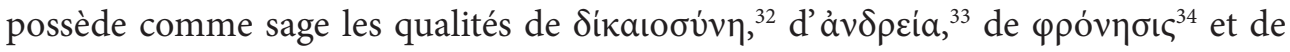
$\sigma \omega \varphi \rho o \sigma u ́ v \eta .{ }^{35}$ Pourtant, ce n'est pas parce qu'on est empereur que l'on est sage. Si la cité est essentiellement constituée d'insensés qu'en est-il de l'Empire? Musonius Rufus a réfléchi à cette question et a proposé que les « rois » philosophent eux aussi. Le

28. Them., Or. 13. Brugisser, 2009, pp. 3-22 ; Konstan, 2009, p. 482. Constantinople est alors perçue

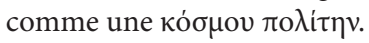

29. Ando, 2007, pp. 429-445 et 2008.

30. Plut., De Alex. fort. 329a-c.

31. Val. Flac., Arg. VII 227-229. Valérius Flaccus, dans son poème épique des Argonautica, au Ir siècle de notre ère, fait dire à Vénus déguisée en Circé s’adressant à Médée que cette dernière doit comprendre que ce monde et les dieux sont communs à toutes les créatures vivantes et que ses limites en sont le lever et le coucher du soleil, ce qui est une façon imagée de parler de l'universel.

32. Muson., VIII 33, 7-14 ; 54, 9-11. Trad. Laurand, 2014, p. 460.

33. Muson., VIII 35, 15-36, 1. Trad. Laurand, 2014, p. 461.

34. Muson., VIII 36, 4-11. Trad. Laurand, 2014, p. 461.

35. Muson., VIII 34, 18-35, 8. Trad. Laurand, 2014, p. 461.

ARYS, 16, 2018 [19-45] ISSN 1575-166X 
roi ou l'empereur doivent être sages, car ils sont la loi vivante qui veille à la légalité et à la concorde. Musonius conseille un "roi », mais ne cherche pas à le guider, à la différence de Sénèque qui cherchait, avec son De clementia, à prendre la direction morale et politique du jeune prince Néron. ${ }^{36} \mathrm{Il}$ mène, en tant que philosophe, une réflexion sur ce que doit être la fonction politique, pourtant considérée comme un indifférent par les stoïciens, au sein de la cité. C'est pourquoi, il faut sans doute interpréter cette option de deux manières chez Musonius. D'une part, il faut être philosophe pour être sage. Sénèque défendit l'idée d'un pouvoir monarchique fort, ${ }^{37}$ vigilant, ${ }^{38}$ consenti et protecteur, ${ }^{39}$ mais il échoua avec Néron sur ce point. Musonius quant à lui distingue exercice du pouvoir royal et « comportement » royal. Plus tard, Marc-Aurèle va assumer la charge impériale comme une mission voulue par la divine providence, alors qu'Épictète n'avait pas proposé de portrait idéal de roi ou de philosophe roi. ${ }^{40} \mathrm{C}^{\prime}$ est pourquoi sans doute Marc-Aurèle fera siens les préceptes de Musonius dans les Pensées où il s'alerte : "Prends garde de te césariser à fond, de t'imprégner (de cet esprit), car c'est ce qui a lieu. Conserve-toi donc simple, honnête, pur, grave, naturel, ami de la justice, pieux, bienveillant, affectueux, ferme dans l'accomplissement des devoirs. Lutte pour demeurer tel que le philosophe a bien voulu te former $»{ }^{41}$

Plus encore, la complexité des relations avec le pouvoir du princeps est représentée par les tensions internes aux membres de l'aristocratie stoïcienne. En effet, Musonius Rufus, rentré d'exil, fit condamner sous Vespasien le sénateur stoïcien Publius Egnatius Celer qui avait faussement accusé auprès de Néron son maitre et patron Quintus Barea Soranus ${ }^{42}$ dont le stoïcisme est discuté. ${ }^{43}$ Cette condamnation fut obtenue, notamment sur la base de la lex Cornelia de falsis contre les faux témoignages étendue par le Senatus consultum Libonianum contre ceux qui avaient touché de l’argent lors des dénonciations. Vespasien qui n'aimait guère Musonius pour avoir voulu prêcher la paix plutôt que la guerre lors de l'année 69 suivit pourtant sa position alors que le Sénat était réticent contre des poursuites envers un de ses membres. De même, un autre stö̈cien, Euphratès, philosophe élève de Musonius, conseilla à Vespasien de rétablir la République, tout en le mettant en garde contre le pythagori-

\footnotetext{
36. Sen., Clem. I 4, 2.

37. Sen., Clem. passim.

38. Sen., Clem. 3, 3.

39. Sen., Clem. 3,$4 ; 4,3 ; 8,6 ; 13,4 ; 14,5 ; 15,5 ;$ Benef. II 20.

40. Epict., Ench. IV 6, 28.

41. M. Aur., Med. VI 30. Trad. Trannoy.

42. PIR ${ }^{2}$ B 55 ; Tac., Hist. IV 10 et 40.

43. Laurand, 2014, pp. 521-523.
} 
cien Apollonios de Tyane et ses manipulations du divin. Cette dénonciation entraîna une défiance tenace des Flaviens envers les conseils parfois contradictoires des philosophes sans qu' il s'agisse d'une hostilité définitive.

Les vagues de persécutions et de condamnations de philosophes montrent qu'il n'y a pas un parallèle absolu entre le statut « royal », la nature « royale » et la sagesse du moins sur le plan politique. L'empereur, dans sa fonction universelle, doit

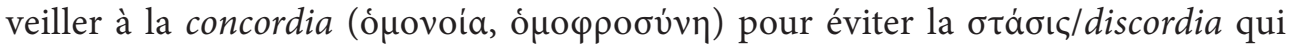

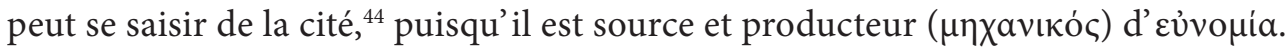
Cette valeur d'abord oligarchique, puisque l'ópovoía supposait une égalité entre les dirigeants devint une valeur monarchique car elle finit par définir le bon exercice du pouvoir par le monarque et la capacité de ce dernier à créer la concorde, à fédérer et donc à intégrer dans un modèle universel les différents corps constitutifs de son empire. Elle devint également, chez les stoïciens, synonyme d'ó $\mu$ o $\lambda$ oүía (conuenientia chez Cicéron). ${ }^{45}$ Cette homologie permit donc de diviniser ó $\mu o v o i ́ a /$ concordia à Rome ${ }^{46}$ et de faire évoluer la notion de concordia vers celle de consensus, son quasi-synonyme. ${ }^{47}$ L'attitude intellectuelle et politique de Cicéron montre d'ailleurs qu'à la fin de la République la notion de concordia ordinum évolue chez l'Arpinate vers une conception plus large qui est celle du consensus omnium bonorum identifiant et homogénéisant tous ceux qui relèvent de la sagesse. L'universalisme se rapproche ici d'un certain cosmopolitisme philosophico-politique. Si le roi ne peut égaler Zeus, le changement d'échelle opéré avec l'Empire rapproche le Princeps diuus de Dieu. On peut d'ailleurs jouer sur un rapport d'échelle. Ainsi, « le même rapport qui unit Dieu au monde unit le roi à l' État, et le roi est à Dieu comme l'État est au monde : car l'État, constitué par l'accordement d'éléments nombreux et divers, imite l'organisation et l' harmonie du monde et le roi, parce qu'il exerce un pouvoir sans contrôle et qu'il est en personne la loi vivante, figure Dieu parmi les hommes $»{ }^{48}$ L'idée de rapport circonscrit des univers différents où le jeu des échelles semble réduire le pouvoir du monarque à sa cité, alors que Dieu règne sur le monde. Toutefois, si on tient compte du changement d'échelle que constitue le pouvoir qu'exerce l'empereur, et la proclamation que son pouvoir s'exerce sur

44. Akar, 2013, pp. 28-36.

45. Cic., Fin. III 21.

46. Cic., Nat. D. II 61.

47. Sur les occurrences de consensus chez Cicéron, voir les notes 153, 154, 155 dans Akar, 2013, pp. 41-42. Pour l'évolution du choix en faveur du terme consensus au détriment de celui de concordia et la rupture chronologique de l'exil de Cicéron en 58 avant notre ère, voir Lepore, 1954, pp. 159-201.

48. Diotogène, 265, 6-12. Trad. Delatte, 1942 citée par Laurand, 2014, p. 516. 


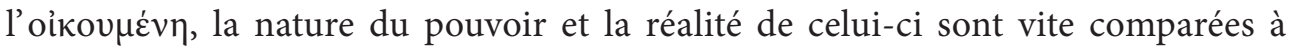
celles de Dieu qui règne sur le monde. L'empereur devient alors le garant de l' har-

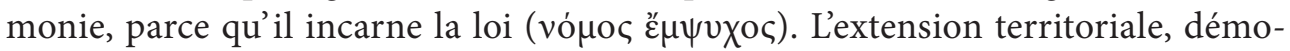
graphique, économique et culturelle modifie le prisme de la conception du pouvoir qui ne semble plus pouvoir s'accommoder du modèle de la cité classique.

Si l'empereur doit être celui qui est le père de l'ensemble des hommes libres de l'empire, la réalité est toutefois plus complexe. Si une communauté des élites se reconnaît sur la base de principes économiques, politiques et culturels, on observe une ségrégation entre citoyens (ciues Romani) et non-citoyens (peregrini, incolae, etc.), ainsi qu'entre Italiens et provinciaux malgré le discours impérial qui s'adresse à tous les hommes où à l'humanité entière. Pline l'Ancien, au milieu du Ir siècle de notre ère confirme toujours la place éminente de l'Italie dans l'empire, parce qu'elle est : « une terre qui est à la fois l'enfant et la mère de toutes les autres, choisie par la volonté des dieux pour donner au ciel même plus d'éclat, rapprocher par la pratique d'une langue commune les idiomes discordants et sauvages de tant de peuples et faire naître le dialogue, donner aux hommes la civilisation, en un mot, devenir l'unique patrie de toutes les nations du monde entier $»^{49}$ On est loin d'une vision de la citoyenneté partagée entre Italiens et provinciaux, bien que l'idéologie augustéenne ait propagé, en même temps que le titre, l'idée du prince pater patriae de ses sujets plutôt que de ses concitoyens comme l'attestent des formules que l'on retrouve en Orient sur certaines inscrip-

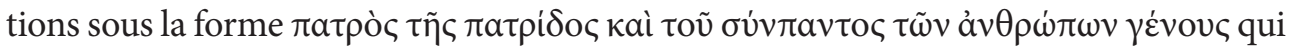
trahit un héritage rhétorique hellénistique parfaitement intégré par les Romains pour qui associer l'ensemble des individus de l'empire dans des formules de communication ne signifie pas penser à une communauté civique homogène dans un commun empire. ${ }^{50}$ D’ailleurs, selon M. Lavan, il ne faut pas exagérer la diffusion de la citoyenneté romaine au cours du Haut-Empire puisqu'à la veille de l'édit de Caracalla il postule que seul un tiers des habitants libres de l'empire formait la communauté des citoyens romains. ${ }^{51}$ Dans la partie orientale, une partie importante des élites municipales et des évergètes n'avait donc pas la citoyenneté romaine et n'en ressentaient peut-être pas le besoin, mais elles se concevaient comme cosmopolites dans le sens où elles se définissaient comme un groupe homogène transcendant les différences ethniques, géographiques et culturelles d'origine. ${ }^{52}$ Lorsque le Sénat peut accueillir ex omnibus prouinciis

49. Plin., HN III 39. Trad. H. Zehnacker. Cf. Verg., Aen. I 282.

50. IGRRP IV, 1756, 101-102. Lavan, 2016b, p. 165.

51. Lavan, 2016a, pp. 3-46.

52. Weisweiler, 2016, p. 189. 
optimates uiros selon les mots prononcés par Nazarius devant le Sénat romain en mars $321,{ }^{53}$ la dimension cosmopolite du Sénat devient possible sans supprimer pour autant les conflits d' intérêts de toutes sortes y compris de " petite patrie ». En s'adressant aux élites avec des formules rhétoriques traditionnelles, le prince comme ses gouverneurs provinciaux pouvait feindre de prendre en compte une communauté dont la langue d'expression et la culture étaient différentes. Si le pouvoir impérial, souhaite une homogénéisation progressive des élites, celle-ci devra passer aussi par la diffusion du latin comme langue administrative et culturelle pour s'imposer aux anciens vaincus qui sont sommés d'adhérer à l'uniformisation culturelle à laquelle conduit l'uniformisation linguistique des élites, singulièrement dans les provinces occidentales mais plus difficilement dans les provinces hellénophones. ${ }^{54}$ C'est à la fin du III ${ }^{\mathrm{e}}$ siècle et au début du $\mathrm{IV}^{\mathrm{e}}$ siècle de notre ère alors que la partie orientale de l'Empire s'individualise de plus en plus que le latin se renforce paradoxalement comme langue administrative et politique, mais aussi comme langue épigraphique qui met en évidence les tendances monarchiques et cosmiques du pouvoir impérial faisant de l'empereur un monarque universel. L'orbis terrarum et le genus humanum lui sont volontairement soumis dans des inscriptions latines qui, à partir de Commode, font apparaître le caractère universel du monarque ${ }^{55}$ qui va s'appuyer sur l'idée de Romanitas dont le sens de concept global apparaîtrait dans nos sources avec Tertullien selon Clifford Ando. ${ }^{56}$ Pourtant, Manilius, contemporain d'Auguste, pensait déjà la cité universelle sur le modèle romain lorsqu' il écrit que « comme, dans le dénombrement des habitants d'une grande ville, on met les sénateurs au premier rang, l'ordre équestre au second, le citoyen après le chevalier, enfin après le citoyen le vil peuple, la populace sans nom ; de même il existe dans le monde une espèce de république établie par la nature, qui du ciel a fait une grande ville. Là, des étoiles représentent les chefs ; d'autres approchent fort près de ces premières : tous les honneurs, tous les droits sont réservés pour ces astres principaux. Le peuple vient ensuite, il est innombrable, il roule au haut de la voûte céleste : si la nature eût accordé à ces petites étoiles des forces proportionnées à leur nombre la région éthérée ne pourrait supporter ses propres feux, et les flammes du ciel embrasé consumeraient l'univers ». ${ }^{57}$

Les débats romains sur le « cosmopolitisme » et l' " universalisme » ont donc été marqués par la dynamique impériale de la conquête territoriale puis de l'intégration des populations vaincues. Avec la diffusion de la ciuitas Romana, Rome fusionne

53. Pan. Lat., IV 35, 1-2.

54. Ando, 2000.

55. Weisweiler, 2016, pp. 196-197.

56. Ando, 2016, p. 179, n. 38.

57. Manilius, Astron. V 730-741. Trad. Nisard.

ARYS, 16, 2018 [19-45] ISSN 1575-166X 
progressivement les différents statuts politiques et s'impose comme le caput mundi unifié. Cette conception repose sur l'interprétation des idées de cosmopolitisme et d'universalisme empruntées à la philosophie grecque et repensées dans une perspective romaine. Les Grecs eux-mêmes vont réviser leur vision du rapport que Grecs et Romains doivent entretenir dans un contexte politique et idéologique profondément transformé. Nombreux vont être les membres des élites grecques qui avaient déjà eu à s'accommoder de la puissance des États hellénistiques à accepter que la réflexion ne s'effectue plus à l'échelle de la cité, mais à celle de l'empire. Le stoïcisme va ainsi proposer des clefs de compréhension et d'acceptation des rapports acceptables entre dominants et dominés réunis dans un monde nouveau où les élites convergent grâce à une même conception du monde..$^{58}$

La converge de l'universalisme et du cosmopolitisme s'appuie sur le dépasse-

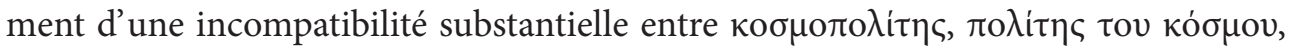

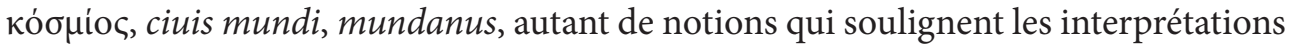
culturelles et diachroniques de concepts grecs revus par les Romains. Ces différentes interprétations ont été parfois en opposition avec les positions fondatrices malgré les précisions et la formalisation conceptuelle de Cicéron ou de Philon d'Alexandrie qui firent du cosmopolitisme une notion philosophique aux implications politiques locales et globales. Une étude lexicale nous montrerait que la notion de kó $\sigma \mu$ íọ renvoie à l'ordre, au bon ordre, à la régularité, à l'acceptation de l'ordre, etc. aussi bien sur les plans moral, politique que religieux, ce qui convient à l'idée que se faisaient les Romains de l'hégémonie qu' ils exerçaient sur leur empire. Ceci dit, les interpré-

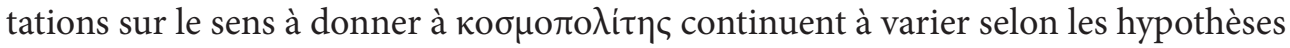
modernes. ${ }^{59}$ D'aucuns s'appuient sur Diogène Laërce ${ }^{60}$ qui rapporte que Diogène le Cynique " avait coutume de dire que les malédictions de la tragédie s'étaient abattues sur lui, qu'en tout cas il était sans cité, sans maison, privé de patrie/mendiant, vagabond, vivant au jour le jour $"{ }^{61}$ Pour d'autres, Diogène le Cynique se fait l'apôtre de la cité lorsqu' il écrit, entre autres syllogismes, que « sans la cité ce qui est moralement beau est inutile; laussi la cité est-elle une réalité moralement belle; /or sans la loi la

58. Shaw, 1985, pp. 37-40.

59. Diog. Laert., VI 63. Trad. M.-O. Goulet-Cazé. Moles, 1995a, pp. 259-280 e 1995b, pp. 120-158 ; Pradeau, 2001, p. 37.

60. Diog. Laert., VI 38. Goulet-Gazé, 1982, pp. 229-231; Shofield, 1999, pp. 141-145; Husson, 2011, pp. 159-163; Chin, 2016, p. 131.

61. Diog. Laert., VI 38 = TrGF 88 F 4 Snell. Trad. M.-O. Goulet-Cazé. 
cité est inutile; / donc la loi est une réalité moralement belle $\gg{ }^{62}$ Cicéron lui-même faisait dire à Balbus, dans le De natura deorum, à propos de la théologie stoïcienne : "Dès lors, il est nécessaire, puisqu'il y a des dieux (dans la mesure où ils existent, ce qui est manifestement le cas), qu'ils soient doués d'une âme, et non seulement d'une âme, mais également en possession de la raison et qu'ils soient liés entre eux par une sorte d'union ou d'association civile, dirigeant un monde unique, comme quelque république et ville commune $"{ }^{63}$ ce qui renforce l'idée stoïcienne d'une cité vraie perçue comme celle du cosmos ; les cités terrestres étant une traduction ou une

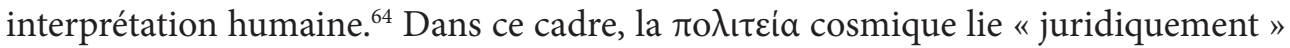

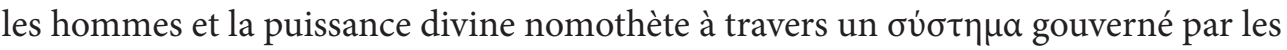
dieux où le sage est destinataire et acteur de la gouvernance divine, organisatrice rationnelle du monde qui exclut les insensés, véritables apatrides ${ }^{65}$ ce que Cicéron avait suggéré dans son De legibus :

« Donc, puisqu' il n'y a rien de mieux que la raison et que celle-ci existe en l' homme et dans la divinité, il y a entre l' homme et la divinité une première association <consistant en une participation> à la raison. Mais pour ceux entre lesquels est commune la faculté de la raison, la droite raison aussi est commune; et, comme c'est elle qui est la Loi, nous devons croire les hommes et les dieux réunis en une association fondée sur la Loi. Or pour ceux qui ont entre eux communauté de loi, il y a communauté de droit. Mais ceux pour qui ces choses (loi et droit) sont communes doivent être considérés comme appartenant à la même Cité. À plus forte raison, s'ils obéissent aux mêmes commandements et aux mêmes autorités, obéissent-ils au mouvement réglé de notre ciel, à la pensée des

62. Diog. Laert., VI 72. Trad. M.-O. Goulet-Cazé. Goulet-Gazé, 1982, pp. 229-231; Dorandi. 1993, pp. 57-68; Husson, 2011, pp. 20-45 ; Helmer, 2017, p. 124 ; et la note complémentaire 5 (pp. 771-772) dans la traduction et les commentaires sur Diogène Laërce, Vies et doctrines des philosophes illustres, Paris, $1999^{2}$ où elle démontre que derrière ce syllogisme se cache une démarche stoïcienne. Moles, 1996, pp. 105-120; Branham, 2007, pp. 71-86.

63. Cic., Nat. D. II 78. Trad. Laurand, 2005, p. 77. Cf. Cic., Fin. III 64. Euseb., Praep. Evang. XV 3-5 = SVF II, 527 et 528.

64. Il est possible que la conformité politique et religieuse du comportement humain avec l'ordre du monde, d'un point de vue que nous pourrions qualifier de "naturaliste ", ait été émise pour la première fois par Héraclite. Dans cette conception, la distinction entre le divin et l' humain n'existe pas en raison de l'osmose que ces deux « états » entretiennent avec l'ordre divin. Héraclite défendait une argumentation à la fois morale et épistémologique en supposant que les hommes doivent se conformer à la nature, c'est-à-dire vivre en conformité avec l'ordonnancement cosmique qui est la loi parfaite. Stob., Eclog. III, 1, 179 ; Sext. Emp., Math. VII 133 Diels-Kranz, Vorsokr. Cf. Pradeau, 2002, p. 175.

65. Shaw, 1985, p. 37 ; Vander Waerdt, 1991, pp. 185-211 ; Laurand, 2005, p. 82.

ARYS, 16, 2018 [19-45] ISSN 1575-166X 
dieux et à la divinité prédominante. Si bien qu'il faut regarder tout cet ensemble du monde comme une Cité unique appartenant en commun aux dieux et aux hommes ».66

Pour autant, les liens entre cité, empire, universalisme et cosmopolitisme restaient complexes et appelaient des précisions que le cadre impérial obligeait à penser. Les interrogations philosophiques, religieuses et politiques liées au statut civique conduisirent, par exemple, Philon d'Alexandrie à revenir à huit reprises sur la question du cosmopolitisme. ${ }^{67}$ Philon qui vécut à Alexandrie les soubresauts qui mirent fin à une relative cohabitation entre communautés grecque et juive en même temps que Rome unifiait sa mainmise impériale sur les territoires du pourtour méditerranéen. Cette uniformisation en voie d'achèvement pose la question du statut des communautés et des individus qui les constituent au sein d'un tout qui est désormais sous le contrôle unique de Rome et de son Princeps. Comment des communautés dont les cultures et les croyances diffèrent vont-elles être incluses dans cet empire ? Philon va reprendre des termes et des concepts stoïciens pour élaborer sur de nombreux points sa propre pensée. ${ }^{68}$ Par exemple, si dans le De Prouindentia, il considère que les grandes mégalopoles sont gouvernées par la providence à l'instar de l'univers, ${ }^{69}$ dans

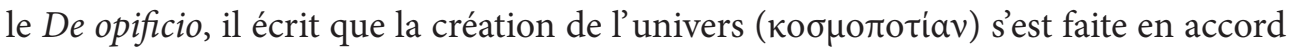
avec la Loi et la Loi en accord avec l'univers ; l'homme qui respecte la Loi est alors

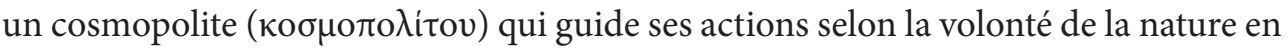

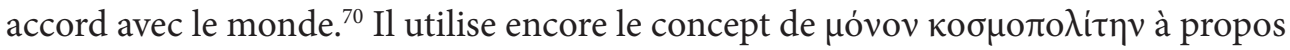
d'Adam qui fut non seulement le premier homme mais aussi le seul cosmopolite à ses yeux. ${ }^{71}$ De même, depuis que les cités ont des constitutions, le cosmopolite se doit de concevoir une constitution qui puisse être commune à tous en la forgeant selon les règles de la nature inspirée par Dieu. ${ }^{72}$ Dans l'esprit de Philon, le cosmopolite est celui qui suit les prescriptions divines selon les règles qui ont présidé à la Genèse. Nous

66. Cic., Leg. I 7, 23. Trad. De Plinval ; Arius Didyme apud Stob., Ecl. II 7, 103, 9W = SVF III, 328.

67. Pour Husson, 2011, p. 159, Philon serait le premier auteur chez qui on trouve l'usage du terme кобноло入ітпс.

68. Ces emprunts ont été parfois mal compris par la critique moderne comme l'atteste l'attribution

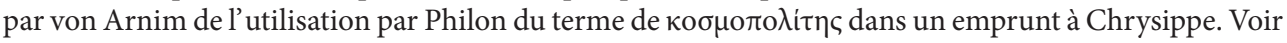
aujourd' hui, Casevitz et Cohen-Skalli, 2019.

69. Pour Clem. Al., Strom. IV 26 les stoïciens pensent que l'univers (ópavóc) est une cite proprement dite, mais que les cités sur terre ne sont pas des cités bien qu’on les appelle ainsi. Schofield, 1999, p. 61; Laurand, 2005, p. 59.

70. Philo, Opif. 3 = SVF III, 336 ; cf. Arius Didyme apud Euseb., Praep. Evang. XV 15, 3-5 = SVF II 528.

71. Philo, Opif. $142=$ SVF III 337.

72. Philo, Opif. $143=$ SVF III 337. 
sommes loin des conceptions de Diogène, de Cicéron ou d'Épictète, car Philon critique ceux qui admirent le monde alors qu' ils devraient, à l' instar de Moïse, un autre

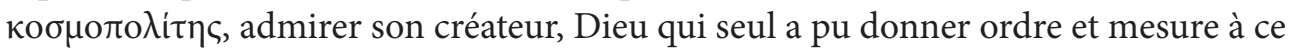
qui est vu comme un monde parfait. C'est pourquoi Moïse est un sage cosmopolite (ò

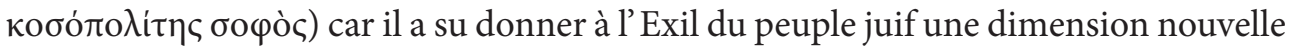
où la fuite se transforme en une quête d'une citoyenneté qui transcende les cités et les États pour se réaliser dans le dessein de Dieu. ${ }^{73}$ On notera que Philon envisage sa réflexion dans le cadre du respect des seules règles mosaïques, alors que les stoïciens

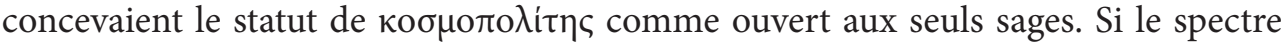
démographique peut sembler plus ouvert, Philon distingue néanmoins trois catégories d'individus : ceux qui dépensent leur vie à la poursuite des plaisirs; ceux qui se consacrent à l'étude et enfin ceux qui sont les hommes de Dieu, prêtres et prophètes qui vivent dans un monde où les contraintes matérielles sont sublimées. Pour lui, les philosophes qui relèvent de la seconde catégorie sont les seuls vrais kosmopolitai. ${ }^{74}$ C'est sans doute dans Des lois spéciales ${ }^{75}$ que Philon donne une définition du cosmopolitisme stoïcien, bien que l'on considère plutôt le De Opificio comme son traité au contenu le plus proche des positions de la Stoa ${ }^{76}$ lorsqu' il aborde les deux principes, actif et passif, ${ }^{77}$ de la création et lorsqu'il reprend l'idée d'une administration de l'univers comme une mégalopolis. Si pour Philon, les hommes sont la reproduction microcosmique du macrocosme qui respecte, avec unité et solidarité, les analogies entre ce qui relève du céleste et ce qui relève du terrestre, l'analogie anthropomorphe fait de l'individu un corps dont les parties sont solidaires à l'instar du Tout. "Ainsi, la raison existant dans l'homme se retrouve dans l'univers régi par la providence. La raison universelle répandue dans la nature n'est autre que le logos divin qui est la loi du monde ${ }^{78}{ }^{78} \mathrm{Il}$ décrit des individus qui observent la nature et tentent de surmonter leurs passions en dotant leurs esprits d'ailes pour faire le tour du monde et mieux l'appréhender comme une cité dont les citoyens partagent une même sagesse fondée sur la vertu et qui leur permet en véritables kosmopolitai de gérer ensemble le gouvernement du monde. Si Philon reconnait chez certains peuples une certaine forme de cosmopolitisme qu'il aborde dans l'un de ses traités les plus stoïciens, le Quod omnis probus liber sit, il ne l'applique pas au peuple juif ni à la communauté

73. Philo, Migr. 59 ; Mos. I 157 ; Conf. 106.

74. Philo, Gig. 61.

75. Philo, Spec. 45.

76. Leisegang, 1941, coll. 1-50 ; Hadas-Lebel, 2003, p. 271.

77. Philo, Opif. 8-9 = SVF II 299-301 et 346a.

78. Hadas-Lebel, 2003, pp. 272-273. Philo, Opif. 143 ; Jos. 29 ; SVF III 316. Lévy, 2017, p. 403.

ARYS, 16, 2018 [19-45] ISSN 1575-166X 
juive d'Alexandrie chez qui le cosmopolitisme juif est celui des Ioudaioi dans le respect des Lois mosaïques. Son cosmopolitisme relève plus de la notion de diaspora ${ }^{79}$ et se définit comme incompatible avec un monde où présiderait le principe impérial romain. Les Ioudaioi « considèrent comme leur métropole la ville sainte où se trouve le temple sacré de Dieu Très Haut, mais ils tiennent pour leurs patries respectives les régions que le sort à données pour séjour à leurs pères, à leurs grands-pères, à leurs arrière-grands-pères et à leurs ancêtres plus lointains encore, où ils sont nés et ont été élevés. Dans certaines, ils sont arrivés en colonie officielle, dès la fondation, pour

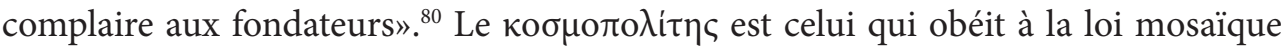
quelle que soit sa patrie d'origine, ce qui empêche les chrétiens d'utiliser cette notion.

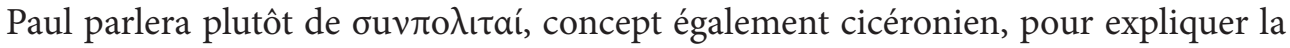
possibilité de tout un chacun d'accéder à Dieu à travers Jésus-Christ. En effet, Paul écrit : "Ainsi, vous n'êtes plus des étrangers, ni des émigrés ; vous êtes concitoyens

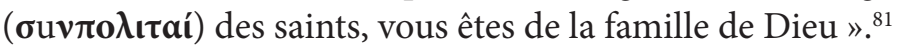

Peut-on conclure que Rome envisagea son empire comme universel et sa citoyenneté comme cosmopolite? Si les hommes et les dieux ont en commun d'appartenir « à la même cité », Jean-François Pradeau précise le périmètre structurel de cette cité commune en suggérant que "l'hypothèse cosmopolitique conjoint

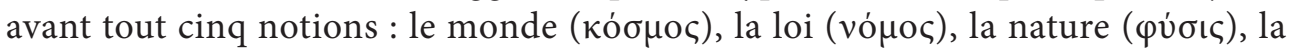

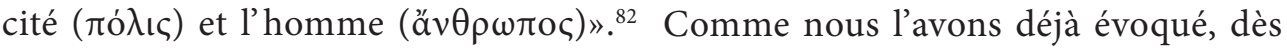
la fin $\mathrm{du} \mathrm{VI}^{\mathrm{e}}$ siècle ou au début du $\mathrm{V}^{\mathrm{e}}$ siècle avant notre ère se pose la question de la naturalité des lois de la cité dans un monde où l'observation fait remarquer qu'il existe des modalités différentes d'organisation selon le cadre géographique, historique, sociologique et religieux qui distinguent les civilisations et renforcent par conséquent la singularité civique, normative et culturelle. ${ }^{83}$ Si les hommes privilégient les institutions locales plutôt que de se conformer à l'ordre cosmique qui est pourtant le même pour tous, c'est parce que les cités terrestres sont le résultat de l'histoire humaine. À Athènes, les sophistes avaient d'ailleurs pensé que les individus pouvaient toujours transgresser ce qui est présenté comme une organisation selon la nature, car comme le formulait Hippias dans le Protagoras

79. Hadas-Lebel, 2003, pp. 55-57.

80. Philo, In Flacc. 46. Trad. Hadas-Lebel, 2003, p. 57.

81. Paul, Eph. 2, 18-19. Trad. Laurand, 2014, p. 438. Goulet-Gazé, 1993, pp. 117-158 ; Husson, 2011, pp. 81-83;

82. Pradeau, 2001, p. 39.

83. Lana, 1951, pp. 196-216. 
de Platon la loi est le tyran de l' homme. ${ }^{84}$ Mais, dans un projet cosmopolitique, il est très tôt convenu que « le sage réglera sa vie de citoyen, non point selon les lois établies, mais selon la loi de la vertu $»{ }^{85}$ dissociant ainsi la raison commune à tous les hommes, l'activité publique et la vertu qui est le propre du sage. Si Diogène a

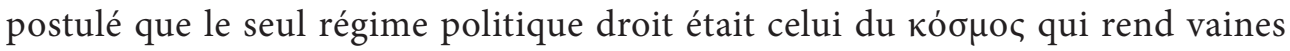
les prétentions de naissance, de gloire et de fortune qui prévalent dans les cités terrestres, il n'y a pas abolition de la cité et de ses lois mais transfert de celles-ci dans le macrocosme qui permet dès lors de faire monde localement, car Diogène qui ne nie pas l'existence des cités et des citoyennetés historiques les critique toutefois. Il pense que l'homme ne peut pas réaliser sa vertu "ici-bas " dans une kallipolis (cité juste). Le cosmopolitisme de Diogène est en quelque sorte

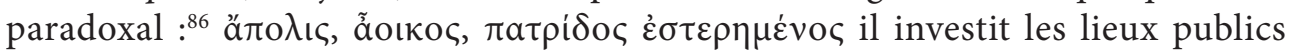
et les occupe non en forme d'habitat et de citoyenneté de substitution mais bien plutôt en tant qu'appropriation civique individuelle d'un espace destiné à tous les citoyens. Pour Philodème, il pensait qu' "aucune cité, aucune loi parmi celles que nous connaissons, n'était à ses yeux une cité ou une loi ${ }^{87}$ et Épictète lui fait dire : «Voyez-moi, je suis sans abri, sans cité (, sans ressource, sans esclaves. Je dors sur la dure. Je n'ai ni femme, ni enfants, ni palais de gouverneur, mais la terre seule et le ciel et un seul vieux manteau. Et qu'est-ce qui me manque ? $\mathrm{Ne}$ suis-je pas sans chagrin et sans crainte, ne suis-je pas libre ? $\gg{ }^{88}$ Enfin, Lucien de Samosate, donne une interprétation sublimée dans le dialogue entre Diogène et son acheteur dans les Sectes à l'ancan ou les Vies de philosophes à vendre ${ }^{89}$ lorsqu' il lui fait répondre à l'Acheteur d'esclaves qui l'interroge sur son origine qu'il est de partout, citoyen du monde ayant pour modèle Héraclès..$^{90}$ En pleine époque antonine, Lucien contourne ainsi la question des origines et de la citoyenneté en faisant de Diogène un homme de partout donc de nulle part. La satire tient dans cette contradiction où un homme libre ne peut être vendu, mais que dire si cet individu ne se reconnaît pas une cité pour origine. Être de partout est-ce nier la

84. Pl., Prt. 337d.

85. Diog. Laert., VI 11 qui cite Antisthène.

86. Arr., Epict. Diss. III 24, 66 : "C'est pourquoi il eut à lui seul la terre entière pour patrie, et aucun pays en particulier ». Trad. Souihlé et Jagu. Cf. Giannantoni, 1990, p. 544 et Husson, 2011, p. 162, n. 1.

87. Phil., De Stoic. XX 3-4 in Dorandi, 1982.

88. Arr., Epict. Diss. III 22, 47-48. Trad. Souihlé et Jagu avec modification de la traduction pour le terme de ä́roגıৎ que nous traduisons par sans cité plutôt que par « sans patrie ». Cf. Laurand, 2014, p. 436.

89. Luc., Biôn prasis 7-11. Chin, 2016, pp. 132-133.

90. Ibid. 8. Husson, 2011, p. 159.

ARYS, 16, 2018 [19-45] ISSN 1575-166X 
cité ? Maxime de Tyr dira de lui qu' « il parcourut la terre, avec l'esprit de l'oiseau, sans craindre de tyran, sans être contraint par la loi ni occupé par la politeia». ${ }^{91}$ Cette forme d'être là où se vit la citoyenneté ne la condamne pas mais au contraire la sublime dans une perspective cynique où la cité appartient aux sages. ${ }^{92}$ C'est sans doute cette conception qui a inspiré les stoïciens et les a incité à développer une philosophie du cosmopolitisme au sein duquel les sages doivent occuper les rouages de décision pour que la " cité " soit bien gouvernée.

En définitive, la diffusion de la citoyenneté romaine ne règle pas le problème substantiel de la coexistence des intérêts particuliers et des intérêts collectifs. Philon d'Alexandrie avait déjà mis les stoïciens devant cette contradiction en soulevant la question des constitutions des cités qui sont aussi singulières que nombreuses et dont le rapport avec la constitution de la nature est conflictuel. On assiste plutôt, selon Philon, à une accumulation des constitutions plutôt qu'à l'uniformisation de

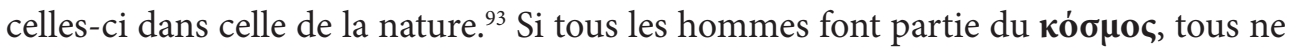
sont pas capables de gouverner. Ce sont les sages qui, ayant cette disposition, l'utilise pour l'intérêt général et l'harmonie ( $\sigma \nu \mu \varphi \omega v i \alpha)$ du monde, ${ }^{94}$ alors que les insensés peuvent s'avérer dangereux car ils ne possèdent pas cette disposition.${ }^{95} \mathrm{Il}$ existe bien une inégalité de fait derrière l'égalité substantielle entre hommes et dieux à propos de la participation à la raison. Seuls les dieux et les sages possèdent la droite raison « car nos natures sont des parties de celle de l'Univers. C'est pourquoi la fin devient : vivre en suivant la nature, c'est-à-dire à la fois la sienne propre et celle de l'Univers, en ne faisant dans nos actions rien de ce qu'a coutume d'interdire la Loi commune, à savoir la Raison droite qui parcourt toutes choses, cette Raison identique à Zeus, qui est, lui, le chef du gouvernement des êtres $»{ }^{96} \mathrm{La}$ participation des sages au gouvernement du monde permet de concevoir le gouvernement de l'Empire comme celui de l'univers par le Princeps et les élites qui philosophent comme le recommande Dion de Pruse, ${ }^{97}$ puisque ces dernières sont, selon Marc-Aurèle, partie ( $\mu \varepsilon \dot{\rho} \rho \varsigma)$ et membre ( $\mu \varepsilon \dot{\lambda} \circ \varsigma)$ de

91. M. Tyr., Philosoph. XXXVI 5 ; cf. Id. XXXII 9.

92. Sur les quatre thèses de la citoyenneté (Cité des Sages, de tous les êtres humains, des hommes et des dieux, des Sages et des dieux). Cf. Vogt, 2012, pp. 65-110.

93. Philo, Jos. 30 = SVF III 323. Laurand, 2014, pp. 478-481.

94. Cic., Leg. I 7, 25 ; Arr., Epict. Diss. II 10, 3-4 ; M. Aur., Med. VII 13.

95. M. Aur., Med. IV 29; VIII 34 et XI 38. Cf. Laurand, 2014, p. 487.

96. Diog. Laert., VII 88. Trad. R. Goulet. Diogène Laërce attribue cette position à Zénon suivi par Cléanthe, Chrysippe, Posidonios et Hécaton.

97. Dio Chrys., Or. 74, 26-27. Reydams-Schils, 2016, pp. 135-140 ; Spinelli, 2016, pp. 150-155. Sur les contradictions on peut aussi consulter Cicéron, Les paradoxes des stoïciens, Plutarque, Contre les stö̈ciens, Sextus Empiricus, Contre les philosophes, etc. cf. Vogt, 2012, pp. 20-64. 
la cité universelle. Il ne s'agit pas de trouver une constitution idéale même si « la constitution politique la meilleure est la constitution mixte, formée de démocratie,

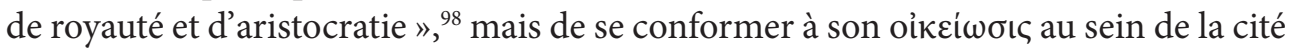
universelle avec laquelle la cité des hommes, dans sa diversité, doit s'articuler, ${ }^{99}$ ce que Cicéron avait incité à faire en "romanisant $»{ }^{100}$ la réalité des deux degrés civiques. En effet, comme on peut être citoyen d'une cité quelconque de l'empire et citoyen romain, on peut être citoyen romain et citoyen du monde. C'est une question de degrés. La constitution antoninienne qui donne la citoyenneté romaine à l'ensemble des hommes libres résidant dans l'empire ne répond pas à ce principe. Elle va plus loin puisqu'elle généralise d'un certain point de vue une série de droits, mais ces derniers ne suffisent pas pour participer à la gestion impériale. Gouverner reste un acte privilégié que seuls ceux qui sont sages peuvent pratiquer.

98. Diog. Laert., VII 131. Trad R. Goulet. Ce passage est sans doute un ajout qui correspond plus à la philosophie de Panétius et de Scipion Émilien comme le laisse supposer Cicéron qui fait dialoguer Laélius et Scipion dans Rep. I 21, 34. Cf. Devine, 1970, pp. 323-336 ; Erskine, 1990, p. 73 ; Laurand, 2005, pp. 144-146; Veillard, 2017, p. 26. Shaw, 1985, p. 28 rappelle que les successeurs de Zénon n’écrivirent pas des traités sur la république mais sur la royauté et la monarchie.

99. M. Aur., Med. IX 29. Lévy, 2017, pp. 378-387.

100. Lévy, 2017, p. 387. 


\section{BibliogRAPHIE}

Africa, T.W. (1959). Stoics, Cynics, and the Spartan Revolution. IRSH, 4, pp. 461-469.

Akar, P. (2013). Concordia. Un idéal de la classe dirigeante romaine à la fin de la République. Paris: Publications de la Sorbonne.

Ando, C. (2000). Imperial Ideology and Provincial Loyalty in the Roman Empire. Berkeley: University of California Press.

Ando, C. (2007). Exporting Roman Religion. In Rüpke, 2007, pp. 429-445.

Ando, C. (2008). The Matter of the Gods: Religion and the Roman Empire. Berkeley: University of California Press.

Ando, C. (2016). Making Romans. Citizens, Subjects, and Subjectivity in Republican Empire. In Lavan, Payne et Weisweiler, 2016, pp. 169-185.

Arruzza, C. et Nikulin, D. (eds.) (2016). Philosophy and Political Power in Antiquity. Leiden et Boston: Brill.

Balot, R.K. (ed.) (2009). A Companion to Greek and Roman Political Thought. Oxford: Wiley-Blackwell.

Becker J.B. (1964). The influence of Roman Stoicism upon the Gracchi's Economic Land Reforms. $P P, 19$, pp. 125-134.

Branham, R.B. (2007). Exile on Main Street. Citizen Diogenes. In Gaertner, 2007, pp. 71-86.

Branham, R.B., Goulet-Cazé, M.-O. (eds.) (1996). The Cynics: The Cynic Movement in Antiquity and Its Legacy. Berkeley: University of California Press.

Brown, E. (2006). Hellenistic Cosmopilitanism. In Gill et Pellegrin, 2006, pp. 549-558.

Brugisser, P. (2009). Numa Pompilius et la Rome sacrée. Regards croisés d'Augustin et de Thémistios. REAug, 55, pp. 3-22.

Brunt, P.A. (1976). The Romanization of the Local Ruling Classes in the Roman Empire. In Pippidi, 1976, pp. 161-174.

Burbank, J. et Cooper, F. (2011). Empires. De la chine ancienne à nos jours (traduction par Christian Jeanmougin de Empires in World History. Power and the Politics of Difference. Paris: Payot.

Casevitz, M. et Cohen-Skalli, A. (2019). Chrysippe, Euvre Philosophique. Paris: Belles Lettres.

Chin, T.T. (2016). What Is Imperial Cosmopolitanism? Revisiting Kosmopolitēs and Mundanus. In Lavan, Payne et Weisweiler, 2016, pp. 129-151.

Delatte, L. (1942). Les traités de la royauté d'Ecphante, Diotogène et Sthénidas. Liège et Paris: Faculté de Philosophie et Lettres et E. Droz.

Devine, F.E. (1970). Stoicism on the best regime. JHI, 31.3, pp. 323-336.

Dorandi, T. (1982). Filodemo. Gli Stoici (P.Herc. 155 e 339). Cronache Ercolanesi, 12, pp. 91 133.

Dorandi, T. (1993). La Politeia de Diogène de Sinope et quelques remarques sur sa pensée politique. In Goulet-Gazé et Goulet, 1993, pp. 57-68.

Dudley, D.R. (1941). Blossius of Cumae. JRS, 31, pp. 94-99.

Erskine, A. (1990). The Hellenistic Stoa, Political Thought and Action. London: Duckworth. 
Finley M.I. (1975a). The Use and Abuse of History. London: Chatto \& Windus.

Finley M.I. (1975b). The Heritage of Isocrates. In Finley, 1975a, pp. 193-214

Gaertner, J.F. (ed.) (2007). Writing Exile. The Discourse of Displacement in Graeco-Roman Antiquity and Beyond. Leiden: Brill.

Giannantoni, G. (1990). Socratis et Socratorum Reliquiae. Napoli: Bibliopolis.

Gill, M.L. et Pellegrin, P. (éds.) (2006). A Companion to Ancient Philosophy. Oxford: Wiley-Blackwell.

Goulet, R. (ed.) (2016). Dictionnaire des philosophes antiques, VI. Paris: CNRS.

Goulet-Cazé, M.-O. (1982). Un syllogisme stoïcien sur la loi dans la doxographie de Diogène le Cynique à propos de Diogène Laërce VI, 72. Rheinisches Museum, 215, pp. 214-240 (repris dans Goulet-Cazé, 2017, pp. 13-33).

Goulet-Cazé, M.-O. (1993). Les premiers cyniques et la religion. In Goulet-Cazé et Goulet, 1993, pp. 117-158 (repris dans Goulet-Cazé, 2017, pp. 421-455).

Goulet-Cazé, M.-O. et Goulet, R. (éds.) (1993). Le Cynisme ancien et ses prolongements. Paris: Presses Universitaires de France.

Goulet-Cazé, M.-O. (éd.) (1999²). Diogène Laërce, Vies et doctrines des philosophes illustres. Paris: Le livre de poche.

Goulet-Cazé, M.-O. (2017). Le cynisme, une philosophie antique, Paris: Vrin.

Gourinat, J.-B. et Barnes, J. (éds.) (2009). Lire les stoïciens. Paris: Presses Universitaires de France.

Hadas-Lebel, M. (2003). Philon d'Alexandrie. Un penseur en diaspora. Paris: Fayard.

Hadot, I. (1970). Tradition stoïcienne et idées politiques au temps des Gracques. REL, 47, pp. 133-179.

Husson, S. (2011). La République de Diogène. Une cité en quête de la Nature. Paris: Vrin.

Helmer, É. (2017). Diogène le Cynique. Paris: Belles Lettres.

Konstan, D. (2009). Cosmopolitan Tradition. In Balot, 2009, pp. 473-484.

Laks, A. et Schofield, M. (éds.) (1995). Justice and Generosity. Cambridge: Cambridge University Press.

Lana, I. (1951). Tracce di dottrine cosmopolitiche in Grecia primo del Cinismo. Rivista di filología classica, 29, pp. 196-216 et 317-338.

Laurand, V. (2005). La politique stö̈cienne. Paris: Presses Universitaires de France.

Laurand, V. (2014). Stö̈cisme et lien social. Enquête autour de Musonius Rufus. Paris: Classiques Garnier.

Lavan, M. (2016a). The Spreed of Roman Citizenship, 14-212 CE: Quantification in the Face of High Uncertainty. Past and Present, 230, pp. 3-46.

Lavan, M. (2016b). "Father of the Whole Human Race". Ecumenical Language and the Limits of Elite Integration in the Early Roman Empire. In Lavan, Payne et Weisweiler, 2016, pp. 153-168.

Lavan, M., Payne, R.E. et Weisweiler, J. (2016). Cosmopolitan Politics. The Assimilation and Subordination of Elite Cultures. In Lavan, Payne et Weisweiler, 2016, pp. 1-28. 
Lavan, M., Payne, R.E. et Weisweiler, J. (éds.) (2016). Cosmopolitanism and Empire. Universal Rulers, Local Elites, and Cultural Integration in the Ancient Near East and Mediterranean. Oxford: Oxford University Press.

Leisegang, H. (1941). S.v. 'Philo'. RE, 20.1, coll. 1-50.

Lepore, E. (1954). Il princeps ciceroniano. Napoli: Istituto italiano per gli studi storici.

Long, A.A. (2008). The Concept of the Cosmopolitan in Greek and Roman Thought. Daedalus, 137,3 , pp. 50-58.

Lévy, C. $\left(2017^{2}\right)$. Cicero Academicus. Recherches sur les Académiques et sur la philosophie cicéronienne. Rome: École Française de Rome.

Moles, J.L. (1995a). Le cosmopolitisme cynique. In Goulet-Gazé et Goulet, 1993, pp. 259-280.

Moles, J.L. (1995b). The Cynics and politics. In Laks et Schofield, 1995, pp. 120-158.

Moles, J.L. (1996). Cynic Cosmopolitism. In Branham et Goulet-Gazé, 1996, pp. 105-120.

Muñiz Grijalvo, E., Cortés Copete, J.M. et Lozano Gómez, F. (éds.) (2017). Empire and Religion. Religious Change in Greek Cities under Roman Rule. Leiden et Boston: Brill.

Nicolet, C. (1965). L'inspiration de Tibérius Gracchus. REL, 67, pp. 142-159.

Pagden, A. (2000). Stoicism, Cosmopolitism, and the Legacy of European Imperialism. Constellations, 7.1, pp. 3-22.

Pippidi, D.M. (éd.) (1976). Assimilation et résistance à la culture gréco-romaine dans le monde ancien. Paris: Belles Lettres.

Pradeau, J.-F. (2001). Imiter l'univers. Remarques sur les origines grecques du cosmopolitisme. Le Télémaque, 19.1, pp. 37-46.

Pradeau, J.-F. (2002). Héraclite, Fragments. Paris: Presses Universitaires de France.

Reydams-Schils, G. (2016). Dio of Prusa and the Roman Stoics on How to Speak the Truth to Oneself and to Power. In Arruzza et Nikulin, 2016, pp. 134-147.

Rüpke, J. (éd.) (2007). A Companion to Roman Religion. Oxford: Wiley-Blackwell.

Schofield, M. (1999²). The Stoic Idea of the City. Chicago: University of Chicago Press.

Shaw Brent, D. (1985). The Divine Economy: Stoicism and Ideology. Latomus, 44.1, pp. 16-54.

Spinelli, E. (2016). Stoic Utopia Reconsidered: Pyrrhonism, Ethics, and Politics. In Arruzza et Nikulin, 2016, pp. 148-163.

Veillard, C. (2017). Les stoïciens étaient-ils démocrates? Cahiers Philosophiques, 151.4, pp. 9-28.

Vogt, K.M. (2012). Law, Reason, and the Cosmic City. Oxford: Oxford University Press. Vander Waerdt, P.A. (1991). Politics and Philosophy in Stoicism. OSAPh, 9, pp. 185-211.

Weisweiler, J. (2016). From Empire to World-State. Ecumenical Language and Cosmopolitan Consciousness in the Late Roman Aristocracy. In Lavan, Payne et Weisweiler, 2016, pp. 187-208. 